\title{
Risk of fracture after bariatric surgery in the United Kingdom: population based, retrospective cohort study
}

\author{
@) $(1) \Theta$ OPEN ACCESS
}

\begin{abstract}
Arief Lalmohamed pharmacoepidemiologist ${ }^{1}$, Frank de Vries assistant professor ${ }^{123}$, Marloes T Bazelier pharmacoepidemiologist ${ }^{1}$, Alun Cooper general practitioner ${ }^{4}$, Tjeerd-Pieter van Staa head of research and honorary professor of epidemiology ${ }^{125}$, Cyrus Cooper director and professor of rheumatology ${ }^{26}$, Nicholas C Harvey senior lecturer and honorary consultant rheumatologist ${ }^{2}$

${ }^{1}$ Utrecht Institute for Pharmaceutical Sciences, Utrecht University, Utrecht, Netherlands; ${ }^{2} \mathrm{MRC}$ Lifecourse Epidemiology Unit, University of Southampton, Southampton General Hospital, Southampton, UK; ${ }^{3}$ Maastricht University Medical Centre, Department of Clinical Pharmacy and Toxicology, Maastricht, Netherlands; ${ }^{4}$ Bridge Medical Centre, Crawley, UK; ${ }^{5}$ General Practice Research Database, Medicines and Healthcare Products Regulatory Agency, London, UK; ${ }^{6}$ Institute of Musculoskeletal Sciences, University of Oxford, Oxford, UK
\end{abstract}

\begin{abstract}
Objectives To estimate fracture risk in patients receiving bariatric surgery versus matched controls.

Design Population based, retrospective cohort study.

Setting Use of records from the United Kingdom General Practice Research Database, now known as the Clinical Practice Research Datalink (from January 1987 to December 2010).

Participants Patients with a body mass index of at least 30 , with a record of bariatric surgery $(n=2079)$, and matched controls without a record $(n=10442)$. Each bariatric surgery patient was matched to up to six controls by age, sex, practice, year, and body mass index. Patients were followed from the date of bariatric surgery for the occurrence of any fracture. We used time dependent Cox regression to calculate relative rates of fracture, adjusted for disease and previous drug treatment, and time-interaction terms to evaluate fracture timing patterns.
\end{abstract}

Main outcome measure Relative rates of any, osteoporotic, and non-osteoporotic fractures.

Results Mean follow-up time was 2.2 years. Overall, there was no significantly increased risk of fracture in patients who underwent bariatric surgery, compared with controls (8.8 v8.2 per 1000 person years; adjusted relative risk $0.89,95 \%$ confidence interval 0.60 to 1.33 ). Bariatric surgery also did not affect risk of osteoporotic and non-osteoporotic fractures. However, we saw a trend towards an increased fracture risk after three to five years following surgery, as well as in patients who had a greater decrease in body mass index after surgery, but this was not significant.

Conclusion Bariatric surgery does not have a significant effect on the risk of fracture. For the first few years after surgery, these results are reassuring for patients undergoing such operations, but do not exclude a more protracted adverse influence on skeletal health in the longer term.

\section{Introduction}

Obesity is an increasing public health problem worldwide. The prevalence of obesity (body mass index $>30$ ), among middle aged Europeans has been estimated as $15-20 \%$. $^{1}$ Data for the prevalence of morbid obesity (body mass index $>40$ ) are lacking in Europe. In the United States, at least $5 \%$ of the population is morbidly obese. ${ }^{2}$ It is now recognised that surgical treatment is the most effective route to weight loss for people with morbid obesity, accompanied by reduction of mortality and improvement of comorbid conditions. ${ }^{3-5}$

Bariatric surgical procedures (conventionally grouped as restrictive or malabsorptive) negatively affect bone remodelling, as suggested by studies on bone resorption markers, and bone mineral density. Restrictive procedures, such as vertical banded gastroplasty and laparoscopic adjustable banding, have been consistently reported to increase bone resorption, ${ }^{6-11}$ an increase that is similar in magnitude to that observed in other forms of weight reduction. ${ }^{8}$ The mechanisms behind the increase in bone resorption after weight loss are not fully understood, but two factors seem to be involved.

Firstly, reduced fat volume may lead to a reduction in circulating concentrations of oestrogens, which are partly synthesised in adipose tissue. ${ }^{10}$ Secondly, a fall in leptin could result in an increase in osteoclast recruitment and bone turnover. ${ }^{12}{ }^{13}$ Malabsorptive procedures such as jejuno-ileal bypass and bilio-pancreatic diversion have also been associated with an increase of bone resorption and a decrease in bone mineral 
density ${ }^{14-19}$; contributory factors clearly include calcium and vitamin D malabsorption, and secondary hyperparathyroidism. ${ }^{20}$ The Roux-en-Y gastric bypass surgery (a combined restrictive and malabsorptive operation) is also associated with increased bone resorption and decreased bone mineral density. ${ }^{1421-27}$

Although evidence indicates that patients may have decreased bone mineral density after bariatric surgery, the effect of the procedure on fracture risk has not been determined. Furthermore, the link between change in body mass index and fracture risk is unknown. Therefore, the aims of this study were to estimate the risk of fracture in patients with bariatric surgery compared with morbidly obese patients who did not undergo surgery, and to quantify the influence of the magnitude of body mass index decrease after surgery on fracture risk.

\section{Methods}

\section{Study population}

A retrospective cohort study was conducted within the General Practice Research Database, now known as the Clinical Practice Research Datalink (www.cprd.com). The Clinical Practice Research Datalink contains computerised medical records of 625 primary care practices in the United Kingdom, representing $8 \%$ of the population. The database provides detailed information on demographics, drug prescriptions, clinical events, specialist referrals, and hospital admissions. Previous studies using the database have shown a high level of data validity with respect to the reporting of fractures ( $>90 \%$ of fractures were confirmed), ${ }^{28}$ and several systematic reviews have reported high degrees of validity and completeness of other diagnoses or smoking status. ${ }^{29-31}$

The study population consisted of all patients with a Clinical Practice Research Datalink read code for bariatric surgery during the period of valid data collection (from January 1987 to December 2010). Gastrointestinal surgery for cancer was excluded in this study, because cancer itself could influence bone metabolism. The index date was defined as the first record for bariatric surgery. Bariatric surgery patients were only included if they had a body mass index record with a value of at least 30 at some point before surgery. Bariatric surgery patients were stratified by surgical technique, including adjustable gastric banding, Roux-en-Y gastric bypass, and other techniques (for example, gastrectomy, and malabsorptive procedures).

\section{Selection of controls}

Each patient was matched by age, sex, body mass index (within a $10 \%$ difference), calendar time, and practice to up to six patients without a history of bariatric surgery (at any time during the study period). Body mass index entries were selected as the latest record before surgery (measured at any time before the index date)

\section{Outcomes}

We followed up patients from the index date to either the end of data collection, the date of transfer of the patient out of the practice area, the patient's death, or fracture (Clinical Practice Research Datalink read codes), whichever came first. Fracture type was stratified according to World Health Organization definitions into osteoporotic fracture (spine, hip, forearm, or humerus) and non-osteoporotic fracture. ${ }^{32}$ For the analyses of these two different fracture groups, we followed up all patients for the occurrence of a fracture in the specific group, regardless of whether a fracture had already occurred in the other group (that is, patients could have sustained both an osteoporotic and non-osteoporotic fracture).

\section{Potential confounders}

General risk factors considered in this study included age, sex, smoking status (a record of currently smoking, ex-smoker, or never smoked before; missing data were treated as a separate category in the analyses), a record of falls in the previous 6-12 months (any fall recorded by the general practitioner; falls in the previous six months were excluded), history of fracture, history of a chronic disease (cerebrovascular disease, heart failure, inflammatory bowel disease, asthma or chronic obstructive pulmonary disease, anaemia, and dementia), and a prescription in the previous six months for glucocorticosteroids, antiobesity drugs, calcium or vitamin D supplements, antihypertensive drugs, loop diuretics, hypnotics or anxiolytics, antipsychotics, antidepressants, proton pump inhibitors, or antiepileptic substances, and drugs for Parkinson's disease treatment. ${ }^{34-37}$ Age and the most recent record of body mass index before the index date were handled as continuous variables in the analyses.

\section{Statistical analysis}

We conducted two main analyses using stratified Cox proportional hazards models (SAS 9.2, PHREG procedure; stratified matched cohort analysis). The first analysis compared the fracture rate in patients with bariatric surgery with that in control patients (with the same body mass index), to yield an estimate of the relative risk of fracture in bariatric surgery patients (stratified by type of fracture and type of bariatric surgical technique). We divided the total follow-up period into 30 day intervals. The presence of risk factors was assessed by reviewing the computerised medical records of risk factors before the start of an interval. We included potential confounders in the final model if they independently changed the $\beta$ coefficient for bariatric surgery by at least $10 \%$.

The second analysis studied the effect of excess loss in body mass index after surgery on fracture risk (with the limit of normality defined as body mass index of 25). For that purpose, we divided all patients with bariatric surgery into four different groups: those with no excess loss after surgery, those with $0-50 \%$ excess loss after surgery, those with at least $50 \%$ excess loss after surgery, and those whose amount of excess loss was unknown. We calculated excess loss as follows:

$100 \times$ (preoperative body mass index-present body mass index $) \div($ preoperative body mass index -25$)$. Based on this excess loss, person time was allocated to one of these four defined categories. In the event of no body mass index assessments in that specific period, the person time was allocated to the category in which excess loss was unknown.

We examined timing of fracture occurrence after bariatric surgery by including time interaction terms (time periodxbariatric surgery) into the model for the following time intervals: less than three months, three to 12 months, one to two years, two to five years, and more than five years. Using smoothing spline regression, ${ }^{38}$ we visualised the time trend for risk of fracture for these given time intervals.

In a sensitivity analysis, we restricted bariatric surgery patients to those with a body mass index record within two months before bariatric surgery, and reset the index date for controls as the date of most recent body mass index recording. These analyses were further adjusted for calendar year and age at the newly defined index date (along with all other confounders). 
Our power analysis demonstrated a power of $88 \%$, assuming a relative risk of 1.6, a type I probability of 0.05 , and based on our cohort sizes (2079 bariatric surgery patients, with an average of 5.02 matched controls per patient, and a fracture probability in the control group of $2.0 \%$ ).

\section{Results}

\section{Baseline characteristics}

Table $1 \Downarrow$ shows baseline characteristics of bariatric surgery patients and matched controls. We identified 2079 patients who underwent bariatric surgery (mean age 44.6 years, $83.9 \%$ female patients, mean body mass index 43.2), and a total of 10442 matched controls (mean age 44.9 years, $85.3 \%$, 40.8). Adjustable gastric banding was the most frequent surgical technique for bariatric surgery $(1249(60 \%))$, followed by Roux-en-Y gastric bypass $(613(29 \%)$, fig $1 \Downarrow)$. The median difference between the index date and most recent record of body mass index was 109 days (interquartile range 241) for patients who underwent bariatric surgery and 321 days (680) for matched controls. Bariatric surgery patients were more likely to have used antidiabetics, antidepressants, anxiolytics or hypnotics, and proton pump inhibitors in the previous six months. Total duration of follow-up was 28899 person years (mean 2.2 years for bariatric surgery patients and 2.3 years for matched controls).

\section{Overall risk of fracture}

Table $2 \Downarrow$ shows the overall risk of fracture in bariatric surgery patients compared with matched controls, stratified by fracture type. We did not observe an increase in overall risk for any fracture $(8.8 v 8.2$ per 1000 person years; adjusted relative risk $0.89,95 \%$ confidence interval 0.60 to 1.33 ), osteoporotic fracture $(0.67,0.34$ to 1.32$)$, or non-osteoporotic fracture $(0.90$, 0.56 to 1.45$)$. Similar rates for any fracture were observed throughout the different surgical techniques.

Figure $2 \Downarrow$ and table $3 \Downarrow$ demonstrate the change in adjusted relative risk with time after surgery, showing a modestly increased risk over the first three months, followed by a reduction and then a trend towards increasing fracture risk after three to five years. However, none of these trends achieved statistical significance, and overall there was no significant interaction between bariatric surgery and time. Our sensitivity analysis showed similar findings when we restricted the sets to bariatric surgery with only recent records of body mass index. Table 2 lists confounders that were included in the final adjusted models.

\section{Risk factors for fracture in bariatric surgery patients}

For bariatric surgery patients, use of anxiolytics in the previous six months (adjusted relative risk 1.82, 95\% confidence interval 1.06 to 3.15$)$, and a history of cerebrovascular disease (8.26, 4.40 to 15.52$)$ or previous fracture $(2.44,1.59$ to 3.76$)$ raised the risk of fracture. Use of antidepressants, antidiabetics, proton pump inhibitors, or statins within six months did not significantly alter fracture risk within these patients (data not shown).

\section{Influence of excess reduction in body mass index after surgery}

Although we saw a trend towards an increased risk of fracture with greater reduction of excess body mass index after surgery, this was not significant (table $4 \Downarrow$ ). However, this analysis had limited statistical power. Thus, compared with patients with a medium excess loss in body mass index (1-50\%), the adjusted relative risk was 0.32 (95\% confidence interval 0.04 to 2.57 ) in those with no excess loss in body mass index, and $1.46(0.55$ to 3.85 ) in those who lost over $50 \%$ of their excess body mass index. The association between body mass index loss and fracture risk remained similar after we included only patients with a body mass index recording in the two months before bariatric surgery.

\section{Discussion}

To our knowledge, this is the first study to investigate fracture risk in patients who underwent bariatric surgery versus matched controls. Although we observed a possible rise in fracture risk at three to five years after surgery, overall, we were not able to demonstrate a significantly increased risk of any,

non-osteoporotic, or osteoporotic fracture with bariatric surgery. We saw a trend towards increasing fracture risk with greater magnitude of excess reduction in body mass index after bariatric surgery, but again, this was not significant.

\section{Comparison with other studies}

Although no fracture studies have compared bariatric surgery patients with matched controls so far, our findings are indirectly supported by a meta-analysis by De Laet and colleagues. ${ }^{39}$ They showed that a decrease in body mass index was less predictive of fracture in obese patients $(>30)$ than in those with a body mass index of less than 30 . For example, when comparing patients with a body mass index of 15 and 20, the researchers found a 3.7-fold elevated risk of hip fracture in the leaner patients. However, when comparing those with a body mass index of 30 and 35, the relative risk was much lower (non-significant 1.1-fold increase in leaner patients). The authors suggested that leanness is a much more important risk factor for fracture, rather than considering obesity as a protective factor. A study by Nakamura and colleagues estimated fracture rates in bariatric surgery patients, but could not compare this group with controls matched by body mass index. ${ }^{40}$ Although they do suggest an increased risk based on expected age and sex specific incidence, this difference may well be the effect of obesity related comorbidities (as we have shown in our baseline characteristics).

So far, studies on bariatric surgery and bone effects have been limited to a number of reports on bone resorption markers and bone mineral density. ${ }^{10-27}$ Although the effect seemed to be small and varied between studies, the results suggested that bariatric surgery might negatively affect bone outcomes. For example, Giusti and colleagues reported a slight decrease in bone mineral density at the femoral neck $(-5.8 \%)$, trochanter $(-6.5 \%)$, but not at the lumbar spine $(+8.0 \%)$, two years after gastric banding procedures. ${ }^{6}$ Similarly, Guney and colleagues showed a $9.9 \%$ drop in bone mineral density at the femoral neck, one year after vertical banded gastroplasty. ${ }^{6}{ }^{10}$ The detrimental effect on bone seemed to be less apparent with malabsorptive procedures. Ten years after biliopancreatic diversion, a $4.2 \%$ decrease in spinal bone mineral density was found, but no significant change in hip bone mineral density. ${ }^{16}$ For the Roux-en-Y gastric bypass, a combined restrictive and malabsorptive procedure, decreases in femoral bone mineral density were found to be as low as $3.5 \%$ after two years, ${ }^{23}$ and as high as $10 \%$ after one year. ${ }^{27}$

The reduction in bone mineral density after bariatric surgery may have several biological mechanisms. Firstly, a fall in bone active adipocyte hormones (oestrogen and leptin) following bariatric surgery may initiate bone loss. Oestrogen depletion has been associated with vertical banded gastroplasty $(22 \%$ 
reduction after one year), ${ }^{10}$ and is strongly linked to bone loss in perimenopausal women. ${ }^{41}$ Decreased leptin levels as a result of weight loss could enhance osteoclast activity and therefore initiate bone loss, ${ }^{12} 13$ and alter the balance between osteoblast and adipocyte formation.

Secondly, lowered levels of insulin and amylin could follow weight loss, resulting in enhanced osteoclast recruitment and inhibition of osteoblast activity. ${ }^{12}$ Thirdly, although evidence is conflicting, malabsorptive procedures could be linked with calcium and vitamin D deficiency (both are associated with a decrease in bone mineral density and increased fracture risk). ${ }^{42}$ Since malabsorptive procedures (including combined restrictive or malabsorptive procedures, such as the Roux-en-Y gastric bypass) are more likely to lead to malnutrition (hypocalcaemia) and vitamin deficiencies than restrictive procedures (for example, gastric banding), ${ }^{43}$ risk of fracture could differ between these surgical techniques. Although limited in statistical power, our study did not observe such a difference in fracture risk between gastric banding and Roux-en-Y gastric bypass. Finally, the effect of bariatric surgery on bone might also depend not only on the type of surgical procedure itself, but also on the degree of sarcopenia caused or accelerated by marked weight loss.

Alternatively, the observed decrease in bone mineral density might be explained by measurement errors of bone mineral density in morbidly obese patients. ${ }^{6}$ Variability of bone mineral density rises substantially when soft tissue depths exceed 25 $\mathrm{cm} .{ }^{44}$ Moreover, Madsen and colleagues showed that fat around bone could falsely increase measured levels of bone mineral density. ${ }^{45}$ As a consequence, reported falls in bone mineral density at femoral and trochanter sites after bariatric surgery could have been overestimated.

\section{Strengths and limitations of the study}

Our study has several strengths. To the best of our knowledge, this is the first cohort of bariatric surgery patients in which the risk of fracture has been investigated. We had a statistical power of $88 \%$ to detect a relative risk of at least 1.6. Our data sources had detailed longitudinal information on drug prescribing and other risk factors for fracture, such as smoking status.

Furthermore, since 2004, body mass index is very well registered within the Clinical Practice Research Datalink (>85\%), which is a result of the introduction of the Quality Outcomes

Framework in 2004. This allowed us to match controls by body mass index accurately, which is important given the association between body weight and bone mineral density. ${ }^{12}$

A major limitation of this study was that body mass index was not routinely collected over short time intervals. We therefore selected the most recent recording of body mass index, assuming this information has not substantially changed over time (before surgery). This lack of data also limited our statistical power in the analysis evaluating the influence of excess reduction in body mass index. Therefore, it was not possible to draw definite conclusions about the role of the magnitude of reduction in body mass index after bariatric surgery. Although obese patients probably change weight continuously, and we did not have information on body mass index at the exact day of bariatric surgery, restricting the study population to those with records in the previous two months did not substantially change the results. Furthermore, the Clinical Practice Research Datalink describes events that occurred or were recorded in general practice. Events occurring in secondary or intermediary services could therefore be incompletely ascertained. In addition, we did not have information on bone mineral density, which could have been useful for determining the underlying biological mechanism in the association between bariatric surgery and fracture.

We cannot exclude the possibility of confounding by (contra)indication in this study. The National Institute for Health and Clinical Excellence guidelines recommend bariatric surgery in morbidly obese patients, preferably with coexisting diseases (for example, type 2 diabetes and hypertension) that could be improved by weight loss. ${ }^{43}$ We did not have information on whether patients were considered for bariatric surgery and then did not undergo an operation because of lack of associated comorbidities. However, since these comorbidities were probably not associated with reduced fracture rate, it is unlikely that this consideration would reduce our ability to detect a difference in fracture rate between bariatric surgery and control patients.

Although a possibility of residual confounding due to unmeasured unbalances between the two study groups still exists, controls in this study seemed to be healthier (with fewer obesity related comorbidities) than patients who underwent bariatric surgery, and could therefore not have masked a true association between bariatric surgery and fracture. Furthermore, poor general fitness (associated with a loss in bone mineral density) may be a reason to not undergo bariatric surgery. Sjöström and colleagues showed that bariatric surgery patients were more physically active than obese controls. ${ }^{46}$ Although we adjusted for factors such as hypertension and use of glucose lowering drugs, we could not adjust for physical activity. However, this healthy user bias would have probably resulted in a decreased fracture risk shortly after surgery, whereas we found a trend towards the opposite. It is usual for patients to modify their diet before surgery to reduce the fat and glycogen content of the liver. This diet may be based on solid or liquid foods. We did not have information on perioperative diet, and therefore were not able to adjust for this potential confounder, but feel that such dietary change over the period of a few weeks would be unlikely to substantially alter fracture risk, particularly because the diet is aimed to preserve muscle tissue.

We used a widely accepted definition of osteoporotic and non-osteoporotic fracture types, but it is difficult to be sure about fracture cause based simply on fracture site, with no information on the level of trauma. Finally, we had a relatively short follow-up time (median time 2.2 years for bariatric surgery patients), which yielded a reduced power to exclude an increase in fracture risk beyond five years.

Contributors: All authors drafted the article, revised it critically for important intellectual content, and approved the final version to be published. CC had full access to all the data in the study and is the study guarantor. All authors were responsible for the study concept and design, and participated in the analysis and interpretation of data. AL led the statistical analysis. $\mathrm{CC}$ and $\mathrm{NCH}$ were responsible for the data acquisition.

Funding: This study was funded by a research grant from the International Osteoporosis Foundation and SERVIER. The funders had no role in study design, data collection and analysis, decision to publish, or preparation of the manuscript.

Competing interests: All authors have completed the Unified Competing Interest form at www.icmje.org/coi_disclosure.pdf (available on request from the corresponding author) and declare: support from the International Osteoporosis Foundation and SERVIER for the submitted work; AL, FV, MB, and TS are employed by the Division of Pharmacoepidemiology and Clinical Pharmacology at Utrecht Institute for Pharmaceutical Sciences, which has received unrestricted research 


\section{What is already known on this topic}

Bariatric surgery can be considered among patients with morbid obesity

Bariatric surgery has been linked to a reduction in bone mineral density, although fracture rates compared with matched controls are unknown

\section{What this study adds}

Bariatric surgery does not have a significant effect on fracture risk

However, there could be an increase in risk after three to five years and in patients who have a greater decrease in body mass index after surgery

funding from the Netherlands Organisation for Health Research and Development, Dutch Health Care Insurance Board, Royal Dutch Pharmacists Association, private-publicly funded Top Institute Pharma (www.tipharma.nl, which includes cofunding from universities, government, and industry), EU Innovative Medicines Initiative, EU 7th Framework Program, Dutch Medicines Evaluation Board, Dutch Ministry of Health and industry (including GlaxoSmithKline, Pfizer); no financial relationships with any organisations that might have an interest in the submitted work in the previous 3 years; no other relationships or activities that could appear to have influenced the submitted work.

Ethical approval: The Clinical Practice Research Datalink group obtained ethical approval from a multicentre research ethics committee for a purely observational research using data from the database, such as ours. This study obtained approval for the independent scientific advisory committee of the Clinical Practice Research Datalink, which is responsible for reviewing protocols for scientific quality.

Data sharing: No additional data available

$1 \quad$ Björntorp P. Obesity. Lancet 1997:350:423-6.

2 Flegal KM, Carroll MD, Ogden CL, Curtin LR. Prevalence and trends in obesity among US adults, 1999-2008. JAMA 2010;303:235-41.

3 Martin LF, Hunter SM, Lauve RM, O'Leary JP. Severe obesity: expensive to society, frustrating to treat, but important to confront. South Med J 1995;88:895-902.

Stunkard AJ. Current views on obesity. Am J Med 1996;100:230-6.

5 Kolanowski J. Surgical treatment for morbid obesity. Br Med Bull 1997:53:433-44.

6 Giusti V, Gasteyger C, Suter M, Heraief E, Gaillard RC, Burckhardt P. Gastric banding induces negative bone remodelling in the absence of secondary hyperparathyroidism: potential role of serum C telopeptides for follow-up. Int J Obes (Lond) 2005;29:1429-35

7 Cundy T, Evans MC, Kay RG, Dowman M, Wattie D, Reid IR. Effects of vertical-banded gastroplasty on bone and mineral metabolism in obese patients. Br J Surg 1996;83:1468-72

8 Olmos JM, Vázquez LA, Amado JA, Hernández JL, González Macías J. Mineral metabolism in obese patients following vertical banded gastroplasty. Obes Surg 2008;18:197-203.

9 Pugnale N, Giusti V, Suter M, Zysset E, Heraief E, Gaillard RC, et al. Bone metabolism and risk of secondary hyperparathyroidism 12 months after gastric banding in obese pre-menopausal women. Int J Obes (Lond) 2003:27:110-6.

10 Guney E, Kisakol G, Ozgen G, Yilmaz C, Yilmaz R, Kabalak T. Effect of weight loss on bone metabolism: comparison of vertical banded gastroplasty and medical intervention. Obes Surg 2003;13:383-8.

11 DiGiorgi M, Daud A, Inabnet WB, Schrope B, Urban-Skuro M, Restuccia N, et al. Markers of bone and calcium metabolism following gastric bypass and laparoscopic adjustable gastric banding. Obes Surg 2008:18:1144-8.

12 Reid IR. Relationships among body mass, its components, and bone. Bone 2002;31:547-55.

13 Meier CA, Bobbioni E, Gabay C, Assimacopoulos-Jeannet F, Golay A, Dayer J-M. IL-1 receptor antagonist serum levels are increased in human obesity: a possible link to the resistance to leptin? J Clin Endocrinol Metab 2002;87:1184-8.

14 Bano G, Rodin DA, Pazianas M, Nussey SS. Reduced bone mineral density after surgical treatment for obesity. Int J Obes (Lond) 1999;23:361-5.

15 Adachi $Y$, Shiota E, Matsumata T, Iso Y, Yoh R, Kitano S. Osteoporosis after gastrectomy: bone mineral density of lumbar spine assessed by dual-energy $\mathrm{X}$-ray absorptiometry. Calcif Tissue Int 2000;66:119-22.

16 Marceau P, Biron S, Lebel S, Marceau S, Hould FS, Simard S, et al. Does bone change after biliopancreatic diversion? J Gastrointest Surg 2002;6:690-8.

17 Newbury L, Dolan K, Hatzifotis M, Low N, Fielding G. Calcium and vitamin D depletion and elevated parathyroid hormone following biliopancreatic diversion. Obes Surg 2003;13:893-5

18 Slater GH, Ren CJ, Siegel N, Williams T, Barr D, Wolfe B, et al. Serum fat-soluble vitamin deficiency and abnormal calcium metabolism after malabsorptive bariatric surgery. $J$ Gastrointest Surg 2004:8:48-55

19 Hamoui N, Kim K, Anthone G, Crookes PF. The significance of elevated levels of parathyroid hormone in patients with morbid obesity before and after bariatric surgery. Arch Surg 2003;138:891-7.

20 Chapin BL, LeMar HJ, Knodel DH, Carter PL. Secondary hyperparathyroidism following biliopancreatic diversion. Arch Surg 1996;131:1048-52.

21 Valderas JP, Velasco S, Solari S, Liberona Y, Viviani P, Maiz A, et al. Increase of bone resorption and the parathyroid hormone in postmenopausal women in the long-term after Roux-en-Y gastric bypass. Obes Surg 2009;19:1132-8.
22 Coates PS, Fernstrom JD, Fernstrom MH, Schauer PR, Greenspan SL. Gastric bypass surgery for morbid obesity leads to an increase in bone turnover and a decrease in bone mass. J Clin Endocrinol Metab 2004;89:1061-5.

23 Von Mach M-A, Stoeckli R, Bilz S, Kraenzlin M, Langer I, Keller U. Changes in bone mineral content after surgical treatment of morbid obesity. Metab Clin Exp 2004;53:918-21.

24 Goode LR, Brolin RE, Chowdhury HA, Shapses SA. Bone and gastric bypass surgery: effects of dietary calcium and vitamin D. Obes Res 2004;12:40-7.

25 Vilarrasa N, Gómez JM, Elio I, Gómez-Vaquero C, Masdevall C, Pujol J, et al. Evaluation of bone disease in morbidly obese women after gastric bypass and risk factors implicated in bone loss. Obes Surg 2009;19:860-6.

26 Gómez JM, Vilarrasa N, Masdevall C, Pujol J, Solano E, Soler J, et al. Regulation of bone mineral density in morbidly obese women: a cross-sectional study in two cohorts before and after bypass surgery. Obes Surg 2009;19:345-50.

27 Johnson JM, Maher JW, Samuel I, Heitshusen D, Doherty C, Downs RW. Effects of gastric bypass procedures on bone mineral density, calcium, parathyroid hormone, and vitamin D. J Gastrointest Surg 2005;9:1106-10.

28 Van Staa TP, Abenhaim L, Cooper C, Zhang B, Leufkens HG. The use of a large pharmacoepidemiological database to study exposure to oral corticosteroids and risk of fractures: validation of study population and results. Pharmacoepidemiol Drug Saf 2000;9:359-66.

29 Herrett E, Thomas SL, Schoonen WM, Smeeth L, Hall AJ. Validation and validity of diagnoses in the General Practice Research Database: a systematic review. Br J Clin Pharmacol 2010;69:4-14.

30 Khan NF, Harrison SE, Rose PW. Validity of diagnostic coding within the General Practice Research Database: a systematic review. Br J Gen Pract 2010;60:e128-36.

31 Lewis JD, Brensinger C. Agreement between GPRD smoking data: a survey of general practitioners and a population-based survey. Pharmacoepidemiol Drug Saf2004;13:437-41.

32 FRAX. WHO Fracture Risk Assessment Tool. 2001. shef.ac.uk. www.shef.ac.uk/FRAX charts.jsp.

33 Lalmohamed A, Welsing PMJ, Lems WF, Jacobs JWG, Kanis JA, Johansson $\mathrm{H}$, et al. Calibration of FRAX $\AA 3.1$ to the Dutch population with data on the epidemiology of hip fractures. Osteoporos Int 2012;23:861-9.

34 Pouwels S, Lalmohamed A, Leufkens B, de Boer A, Cooper C, van Staa T, et al. Risk of hip/femur fracture after stroke: a population-based case-control study. Stroke 2009;40:3281-5.

35 Pouwels S, Lalmohamed A, Souverein P, Cooper C, Veldt BJ, Leufkens HG, et al. Use of proton pump inhibitors and risk of hip/femur fracture: a population-based case-control study. Osteoporos Int 2011;22:903-10.

36 Pouwels S, Van Staa TP, Egberts ACG, Leufkens HGM, Cooper C, De Vries F. Antipsychotic use and the risk of hip/femur fracture: a population-based case-control study. Osteoporos Int 2009;20:1499-506.

37 De Vries F, Pouwels S, Lammers JWJ, Leufkens HGM, Bracke M, Cooper C, et al. Use of inhaled and oral glucocorticoids, severity of inflammatory disease and risk of hip/femur fracture: a population-based case-control study. J Intern Med 2007;261:170-7.

38 Lalmohamed A, Vestergaard P, Klop C, Grove EL, de Boer A, Leufkens HG, et al. Timing of acute myocardial infarction in patients undergoing total hip or knee replacement: a nationwide cohort study. Arch Intern Med 2012, 23 July, doi:10.1001/archinternmed.2012. 2713.

39 De Laet C, Kanis JA, Odén A, Johanson H, Johnell O, Delmas P, et al. Body mass index as a predictor of fracture risk: a meta-analysis. Osteoporos Int 2005;16:1330-8.

40 Nakamura K, Haglind E, Clowes J, Achenbach S, Atkinson E, Melton LJ, et al. ENDO conference abstract: fracture risk after bariatric surgery. ENDO Conference, 2009.

41 Recker R, Lappe J, Davies K, Heaney R. Characterization of perimenopausal bone loss: a prospective study. J Bone Miner Res 2000;15:1965-73.

42 Viégas M, Vasconcelos RS de, Neves AP, Diniz ET, Bandeira F. Bariatric surgery and bone metabolism: a systematic review. Arq Bras Endocrinol Metabol 2010;54:158-63.

43 Leff DR, Heath D. Surgery for obesity in adulthood. BMJ 2009;339:b3402.

44 Van Loan MD, Johnson HL, Barbieri TF. Effect of weight loss on bone mineral conten and bone mineral density in obese women. Am J Clin Nutr 1998;67:734-8.

45 Madsen OR, Jensen JE, Sørensen OH. Validation of a dual energy X-ray absorptiometer: measurement of bone mass and soft tissue composition. Eur J Appl Physiol Occup Physiol 1997;75:554-8.

46 Sjöström L, Lindroos A, Peltonen M, Torgerson J, Bouchard C, Carlsson B, et al. Lifestyle, diabetes, and cardiovascular risk factors 10 years after bariatric surgery. $N$ Eng $J$ Med 2004;351:2683-93.

Accepted: 06 July 2012

\section{Cite this as: BMJ 2012;345:e5085}

This is an open-access article distributed under the terms of the Creative Commons Attribution Non-commercial License, which permits use, distribution, and reproduction in any medium, provided the original work is properly cited, the use is non commercial and is otherwise in compliance with the license. See: http://creativecommons.org/licenses/bync/2.0/ and http://creativecommons.org/licenses/by-nc/2.0/legalcode. 


\section{Tables}

Table 1/ Baseline characteristics of bariatric surgery patients and controls matched by age, sex, and body mass index. Data are no (\%) of patients unless stated otherwise

\begin{tabular}{|c|c|c|}
\hline Characteristic & Bariatric surgery patients ( $n=2079$ ) & Matched controls ( $n=10442)$ \\
\hline Follow-up time (years) ${ }^{*}$ & $2.2(2.1)$ & $2.3(2.2)$ \\
\hline Female patients & $1744(83.9)$ & $8904(85.3)$ \\
\hline Age at index date (years) ${ }^{\star}$ & $44.6(11.1)$ & $44.9(11.2)$ \\
\hline Body mass index at index date ${ }^{*}$ & $43.2(7.2)$ & $40.8(6.4)$ \\
\hline \multicolumn{3}{|l|}{ Smoking status } \\
\hline Never & $1092(52.5)$ & $5957(57.0)$ \\
\hline Current & $377(18.1)$ & $2293(22.0)$ \\
\hline Ex-smoker & $591(28.4)$ & $2100(20.1)$ \\
\hline Unknown & $19(0.9)$ & $92(0.9)$ \\
\hline Falls (within previous 6-12 months) & $22(1.1)$ & $58(0.6)$ \\
\hline \multicolumn{3}{|l|}{ History of disease ever before } \\
\hline Fracture & $407(19.6)$ & $1876(18.0)$ \\
\hline Rheumatoid arthritis & $30(1.4)$ & $120(1.1)$ \\
\hline Cerebrovascular disease & $37(1.8)$ & $156(1.5)$ \\
\hline \multicolumn{3}{|l|}{ Drug use within previous 6 months } \\
\hline Glucocorticoids & $89(4.3)$ & $370(3.5)$ \\
\hline Calcium or vitamin D supplements & $104(5.0)$ & $174(1.7)$ \\
\hline Antiobesity drugs & $248(11.9)$ & $576(5.5)$ \\
\hline Antidiabetics & $401(19.3)$ & $1102(10.6)$ \\
\hline Antidepressants & $697(33.5)$ & $2050(19.6)$ \\
\hline Anxiolytics or hypnotics & $203(9.8)$ & $574(5.5)$ \\
\hline Bisphosphonates & $8(0.4)$ & $68(0.7)$ \\
\hline Hormone replacement therapy & $90(4.3)$ & $271(2.6)$ \\
\hline Proton pump inhibitors & $503(24.2)$ & $1315(12.6)$ \\
\hline
\end{tabular}

*Data are mean (standard deviation). 
Table 2| Risk of fracture in bariatric surgery patients compared with controls matched by age, sex, and body mass index, by type of fracture

\begin{tabular}{lccc} 
& Fracture $(\mathbf{n o}(\%))$ & Crude relative risk (95\% $\mathbf{C l})^{*}$ & Adjusted relative risk (95\% Cl)t \\
No bariatric surgery & $207(2.0)$ & Reference & $0.89(0.60$ to 1.33$)$ \\
\hline Any fracture after bariatric surgery & $38(1.8)$ & $1.07(0.74$ to 1.54$)$ & $0.82(0.50$ to 1.36$)$ \\
\hline Type of bariatric surgery & & $0.85(0.53$ to 1.36$)$ & $0.77(0.27$ to 2.16$)$ \\
\hline Gastric banding & $21(1.0)$ & $2.11(0.98$ to 4.56$)$ & $1.28(0.42$ to 3.92$)$ \\
\hline Roux-en-Y gastric bypass & $9(0.4)$ & $1.24(0.51$ to 3.01$)$ & \\
\hline Other & $8(0.4)$ & & $0.90(0.56$ to 1.45$)$ \\
\hline Type of fracture & & $1.01(0.65$ to 1.57$)$ & $0.67(0.34$ to 1.32$)$ \\
\hline Non-osteoporotic & $26(1.3)$ & $0.88(0.47$ to 1.64$)$ & \\
\hline Osteoporotic & $13(0.6)$ & &
\end{tabular}

*Adjusted for age, sex, and most recent record of body mass index before the index date.

†Adjusted for age, sex, and most recent record of body mass index before the index date; a history of fracture, inflammatory bowel disease, and cerebrovascular disease ever before; a history of falls in the previous 6-12 months; and use of glucocorticoids, calcium or vitamin D supplements, antiobesity drugs, antihypertensive drugs, loop diuretics, organic nitrates, antidepressants, anxiolytics or hypnotics, bisphosphonates, opioids (tramadol or stronger), and proton pump inhibitors in the previous six months. 
Table 3| Risk of any fracture in bariatric surgery patients and matched controls over time Bariatric surgery patients

Matched controls

Time since index date (months) No at risk No of events No of person years Rate (\%)* No at risk No of events No of person years Rate (\%)*

\begin{tabular}{lccccccccc}
$0-3$ & 2079 & 5 & 493 & 1.01 & 10442 & 20 & 2619 & 0.76 \\
\hline $4-12$ & 1916 & 12 & 1225 & 0.98 & 9733 & 55 & 6622 & 0.83 \\
\hline $13-24$ & 1393 & 9 & 1119 & 0.80 & 7255 & 49 & 6191 & 0.79 \\
\hline $25-60$ & 888 & 8 & 1357 & 0.59 & 4717 & 63 & 7577 & 0.83 \\
\hline$>60$ & 170 & 4 & 337 & 1.19 & 1003 & 20 & 2200
\end{tabular}

${ }^{*}$ Calculated as the number of events divided by the number of person years in each period. 
Table 4| Risk of any fracture in bariatric surgery patients, by excess body mass index change during follow-up

Any fracture (no (\%)) Crude relative risk $(95 \% \mathrm{Cl})^{*}$ Adjusted relative risk $(95 \% \mathrm{Cl}) \dagger$

Any fracture after bariatric surgery $38(1.8)$

Excess loss in body mass index

\begin{tabular}{lccc}
\hline$<0 \%$ & $1(0.0)$ & $0.31(0.04$ to 2.49$)$ & 0.32 (0.04 to 2.57$)$ \\
\hline $0-50 \%$ & $9(0.4)$ & Reference & Reference \\
\hline$>50 \%$ & $8(0.4)$ & $1.58(0.61$ to 4.11$)$ & $1.46(0.55$ to 3.85$)$ \\
\hline Unknown & $20(1.0)$ & $0.56(0.25$ to 1.25$)$ & $0.51(0.23$ to 1.15$)$ \\
\hline
\end{tabular}

*Adjusted for age, sex, and most recent record of body mass index before the index date.

†Adjusted for confounders as shown in table 2. 


\section{Figures}

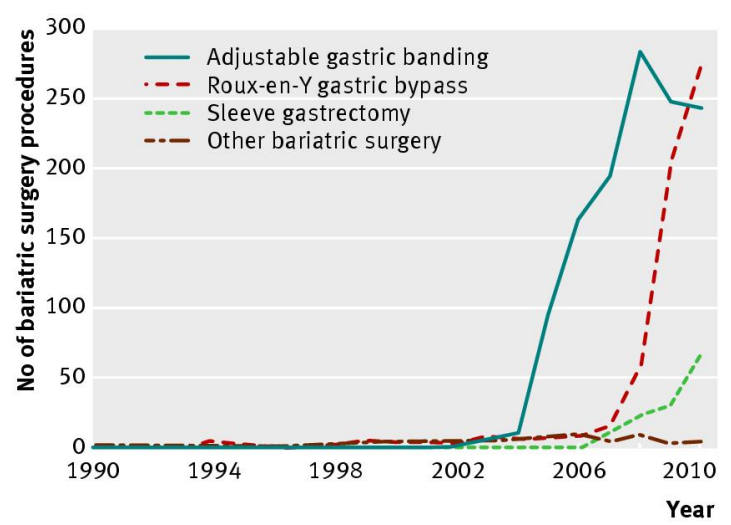

Fig 1 Number of bariatric surgery procedures performed between 1990 and 2010, by year and type of bariatric surgery

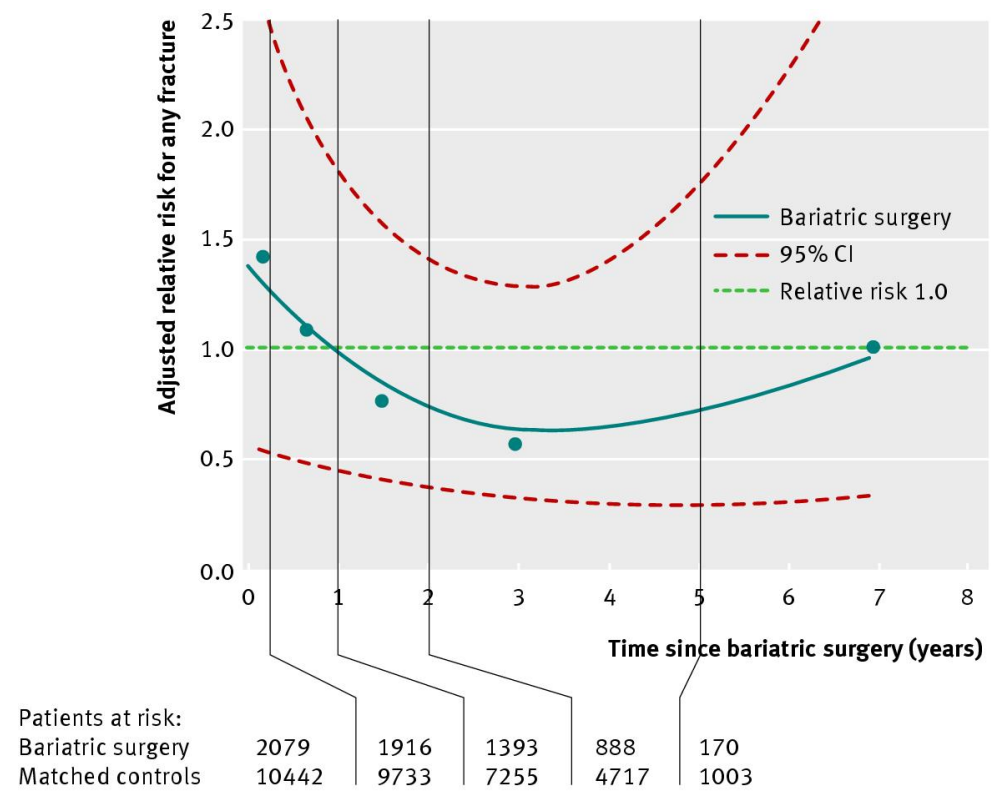

Fig 2 Spline regression plot of time since bariatric surgery and risk of any fracture in bariatric surgery patients versus matched controls. Risk adjusted for confounders as shown in table 2 